\title{
Erratum: Monitoring plant functional diversity from space
}

Walter Jetz, Jeannine Cavender-Bares, Ryan Pavlick, David Schimel, Frank W. Davis, Gregory P. Asner, Robert Guralnick, Jens Kattge, Andrew M. Latimer, Paul Moorcroft, Michael E. Schaepman, Mark P. Schildhauer, Fabian D. Schneider, Franziska Schrodt, Ulrike Stahl and Susan L. Ustin

Nature Plants 2, 16024 (2016); published online 2 March 2016; corrected 14 March 2016

A Supplementary Information file was inadvertently omitted from the Comment when originally published; this has now been rectified. 